\title{
BMJ Open Optimal timing for elective caesarean delivery in a Chinese population: a large hospital-based retrospective cohort study in Shanghai
}

\author{
Yong HU, ${ }^{1}$ Hong Shen, ${ }^{2}$ Mark B Landon, ${ }^{3}$ Weiwei Cheng, ${ }^{2}$ Xiaohua Liu ${ }^{2}$
}

To cite: HU Y, Shen $\mathrm{H}$, Landon MB, et al. Optimal timing for elective caesarean delivery in a Chinese population: a large hospitalbased retrospective cohort study in Shanghai. BMJ Open 2017;7:e014659. doi:10.1136/ bmjopen-2016-014659

- Prepublication history and additional material are available. To view these files please visit the journal online (http://dx.doi org/10.1136/bmjopen-2016014659).

YHU and HS contributed equally.

Received 10 0ctober 2016 Revised 21 February 2017 Accepted 24 February 2017

\section{CrossMark}

${ }^{1}$ Department of Neonatology, Shanghai Children's hospital, Shanghai Jiaotong University, Shanghai, China

${ }^{2}$ Obstetrics Department, International Peace Maternity \& Child Health Hospital, Shanghai Jiaotong University, Shanghai, China

${ }^{3}$ Division of Maternal Fetal Medicine, Department of Obstetrics \& Gynecology, The Ohio State University College of Medicine, Columbus, Ohio, USA

Correspondence to

Dr Xiaohua Liu; annaabcd114@ hotmail.com

\section{ABSTRACT}

Objective To assess the relationship between the timing of antepartum elective caesarean delivery (CD) at term and perinatal outcomes in a Chinese population.

Methods We conducted a retrospective cohort study of mode of delivery at a large obstetric centre in Shanghai, China between 2007 and 2014. Eligibility criteria included: term nulliparous women with a singleton gestation undergoing antepartum elective CD.

Results There were 19939 women delivered by antepartum CD without indications, with $5.9 \%$ performed at 37-37 6/7 weeks, $36.2 \%$ at 38-38 6/7 weeks, $38.4 \%$ at 39-39 6/7 weeks, $15.4 \%$ at $40-406 / 7$ weeks, $4.0 \%$ at $\geq 41$ weeks. As compared with births at 39-39 6/7 weeks, births at 37 weeks were associated with an increased odds of neonatal respiratory disease (adjusted odds ratian(aOR): $4.82 ; 95 \% \mathrm{Cl} 3.35$ to 6.94 ), neonatal infection (aOR: $3.68 ; 95 \% \mathrm{Cl} 1.80$ to 7.52 ), hypoglycaemia (a0R: $3.85 ; 95 \% \mathrm{Cl} 2.29$ to 6.48), hyperbilirubinaemia (a0R: 3.50; 95\% $\mathrm{Cl} 2.12$ to 5.68), neonatal intensive care admission (aOR: 3.73; 95\% $\mathrm{Cl} 2.84$ to 4.89) and prolonged hospitalisation (aOR: $7.51 ; 95 \% \mathrm{Cl} 5.10$ to 11.07 ). Births at 38 weeks, 40 weeks or $\geq 41$ weeks were also associated with an increased odds of neonatal respiratory disease with corresponding aORs $(95 \% \mathrm{Cl})$ of 2.26 (1.71 to 3.00$)$, 1.97 (1.33 to 2.94) and 2.91 (1.80 to 4.70), respectively. Conclusion For women undergoing elective $\mathrm{CD}$, neonatal outcome data suggest that delivery at 39-39 6/7 complete weeks is optimal timing in a Chinese population.

\section{INTRODUCTION}

Infants delivered <39 complete gestational weeks are believed to be at increased risk for neonatal adverse respiratory outcomes and a composite adverse neonatal outcome. The risk increases gradually as gestational week at birth declines, especially when the infants are delivered by antepartum caesarean section without labour. ${ }^{1-4}$ As a result, national clinical practice guidelines in the UK, USA and Canada recommend that planned caesareans should not be performed before 39 gestational weeks without specific indications. ${ }^{5-7}$ However, neonatal outcomes based on gestational age have been reported

\section{Strengths and limitations of this study}

This is a large hospital-based retrospective cohort study which had been reported several times previously. This large population with elective primary caesarean delivery (CD) without indication provides a unique opportunity to determine the optimal timing in relation to neonatal outcomes.

- An important concern for optimal timing of elective delivery at term is the ongoing risk of stillbirth with increasing gestational weeks. In previous observational studies concerning optimal timing of elective caesarean section, the stillbirth rate was not evaluated. In our study, we were able to calculate the stillbirth rates per 1000 ongoing pregnancies at each particular gestational week in the entire cohort.

- The study population had a low body mass index and was very homogeneous ( $99 \%$ Han) which may limit its generalisability to other populations.

- There were only five neonatal deaths and one intrapartum stillbirth despite the large population studied. Thus our study was underpowered to analyse the timing of $\mathrm{CD}$ in relation to these serious perinatal outcomes.

varies in different race.,. Some studies have suggested that the advantages of waiting until 39 weeks to perform planned caesarean delivery (CD) for white women may not be evident in South Asians. ${ }^{89}$ Without the direct evidence from a Chinese population study, the guideline of waiting to 39 weeks has not been formally implemented in China. The data concerning timing of elective caesarean section have largely come from women undergoing repeat procedures. ${ }^{14}$ Only few studies have included a small proportion of primary procedures. $^{23}$

China has the highest $\mathrm{CD}$ rate in the world. A major reason for the high $\mathrm{CD}$ rate is the large proportion of elective $\mathrm{CD}$ on maternal request (CDMR). ${ }^{10-13}$ 'None indication' is actually the most common indication for CD in developed areas of China. Many factors, 
including the parents' preference of a specific day such as a birth day and physician convenience, have contributed to CD before 39 gestational weeks. ${ }^{10}$ There is also increasing enthusiasm for CDMR with a rate of $2.5 \% \sim 4 \%$ in the western countries. ${ }^{14}$ The optimal timing of primary elective $\mathrm{CD}$ is of important public health implications. Therefore, we undertook the present study of a large, retrospective cohort of women to assess the relationship between gestational age at delivery and the risk of adverse perinatal outcomes in a Chinese nulliparous population.

\section{MATERIALS AND METHODS \\ Study design}

We examined the discharge data of all deliveries $\geq 24$ gestational weeks at the International Peace Maternity \& Child Healthcare Hospital (IPMCHH), Shanghai Jiaotong University from 2007 through 2014. Our institute is one of the largest maternity centres in Shanghai, with over 12000 annual deliveries and over 90\% women being nulliparous due to the Chinese one-child policy which ended in 2015.

\section{Study cohort}

Eligibility criteria for the present cohort included: nulliparous women with singletons who delivered at term. Those with major fetal defects were excluded from the cohort.

\section{Data collection}

We used the same data set which had been reported previously. ${ }^{15}$ In brief, data were extracted and abstracted from IPMCHH electronic medical records. The abstracted information included maternal demographic characteristics (such as age, insurance, body mass index, etc), reproductive and prenatal history, chronic medical history, labour and delivery summaries, and postpartum and infant information. The anonymous data were then put into a standardised collection form prior to analysis.

Antepartum elective or non-indicated CD was defined as an antepartum caesarean section performed either on maternal request or physician preference without medical indications which has been described previously. ${ }^{10}$ Cases of antepartum elective or non-indicated CD could be identified in this study as IPMCHH requires that a signed patient consent form outlining the risks and benefits of $\mathrm{CD}$ be retained in the medical record. The timing of delivery was determined in completed weeks of gestation such that 37 weeks, for example, included deliveries at 37 0/7-37 6/7 weeks. Gestational age was based on the combination of last menstrual period and first-trimester ultrasound.

The stillbirth rates per 1000 ongoing pregnancies were calculated in the whole cohort of 81507 eligible women. The following neonatal outcomes calculated in the antepartum non-indicated CD group were studied: neonatal mortality at less than 28 days, respiratory complications (registered as respiratory distress syndrome (RDS), transient tachypnoea of the newborn, pneumothorax), hypoglycaemia, necrotising enterocolitis, hypoxic-ischaemic encephalopathy, meconium aspiration syndrome, neonatal infection, hyperbilirubinaemia, the neonatal intensive care unit (NICU) admission and prolonged neonatal hospitalisation ( 5 days or longer). The diagnosis criteria of RDS included: respiratory distress, radiological features, and oxygen treatment with a fraction of inspired oxygen $\geq 0.40$ for at least 24 hours or until death. The neonatal transient tachypnoea was diagnosed by the presence of tachypnoea within hours after delivery and typical radiological features. The neonatal hypoglycaemia was defined by a plasma glucose level of $<1.9 \mathrm{mmol} / \mathrm{L}$ or the need of therapy with intravenous glucose. Neonatal infection included pneumonia, sepsis, meningitis or antibiotic management for 3 days or more.

The standardised protocol of the antenatal fetal testing and obstetric management in low-risk pregnancies in Shanghai includes: (1) Non-stress testing at 36 gestational weeks, and weekly thereafter; (2) Ultrasound fetal measurement with biophysical profile scores is routinely performed at 38 weeks; (3) Obstetricians make a delivery plan with women at 37 38 weeks.

SAS software, V.9.2 (SAS Institute, Cary, North Carolina, USA) was used to analyse data. Descriptive statistics included numbers and frequencies for categorical variables, means and SD for continuous variables. The incidence of adverse maternal and infant outcomes was calculated for each gestational week at the time of $\mathrm{CD}$. Trends in the incidence rates of adverse neonatal outcomes were assessed by the Cochran-Armitage test. Logistic regression was used to assess adjusted odds ratio $(\mathrm{aOR}) \mathrm{s}$ for the association between gestational age at birth and neonatal outcomes relative to 39 gestational weeks. Maternal age, education, insurance status, prepregnancy body mass index, type of conception, pregnancy complications and maternal chronic medical conditions were included as confounders in the logistic models. A nominal two-sided $\mathrm{p}$ value of $<0.05$ was considered statistically significant.

\section{RESULTS}

We extracted and abstracted data of 98892 pregnancies to 95603 unique women who were delivered at IPMCHH through 2007-2014. After restricting the sample to inclusion criteria, we were left with 81507 (82.4\%, including 48 stillbirths) women for analysis. There were 48 stillbirths after $37^{+0}$ gestational weeks in this cohort. At 37, 38, 39, 40, 41 gestational weeks, the stillbirth rates per 1000 ongoing pregnancies were $0.20,0.20,0.26,0.07$ and 0.16 , respectively ( $\mathrm{p}$ for gestational weeks trend $<0.05$ ) (table 1 ). In the other 81459 women with a live fetus, there were 50912 women who attempted vaginal delivery (spontaneous vaginal birth, assisted vaginal delivery or intrapartum CD) and 10608 antepartum CD with indications. We identified 19939 women delivered by antepartum elective or non-indicated CD (figure 1). Among the women who underwent antepartum non-indicated CD at term, 5.9\% 
Table 1 The stillbirth rates per 1000 ongoing pregnancies

\begin{tabular}{clll}
\hline $\begin{array}{l}\text { Gestational } \\
\text { week }\end{array}$ & Stillbirths (n) & $\begin{array}{l}\text { Ongoing } \\
\text { pregnancies }\end{array}$ & $\begin{array}{l}\text { Rate*(per } \\
\text { 1000) }\end{array}$ \\
\hline 37 & 16 & 81507 & 0.20 \\
38 & 15 & 75276 & 0.20 \\
39 & 14 & 54273 & 0.26 \\
40 & 2 & 26716 & 0.07 \\
$\geq 41$ & 1 & 6176 & 0.16 \\
\hline
\end{tabular}

*Rate is stillbirths per 1000 ongoing pregnancies or 'fetuses at risk' at the gestational week.

underwent the procedure at $37-376 / 7$ weeks, $36.2 \%$ at $38-386 / 7$ weeks, $38.4 \%$ at $39-396 / 7$ weeks, $15.4 \%$ at $40-406 / 7$ weeks and $4.0 \%$ at $\geq 41$ weeks. Thus, $42.1 \%$ antepartum non-indicated CDs were performed before 39 gestational weeks.

More than $99 \%$ of the women in our present cohort were of Han ethnicity. Baseline and obstetric characteristics of the study population are shown in table 2. Gestational age was confirmed by a first- trimester ultrasound examination in $94.2 \%$ of pregnancies. Older ( $\geq 35$ years old), obese women or women complicated with coexisting medical disorders were more likely to undergo $\mathrm{CD}$ prior to 39 gestational weeks $(\mathrm{p}<0.001)$. Women with male fetuses or those conceived after assisted reproduction were also more likely to undergo CD prior to 39 weeks $(p<0.001)$. Conversely, women with medical insurance were less likely to undergo $\mathrm{CD}$ prior to 39 gestational weeks. The birth weight of the newborns and the prevalence of macrosomia $(\geq 4000 \mathrm{~g})$ increased progressively with greater gestational age at CD.

Figure 2 and table 3 show the relationship between timing of $\mathrm{CD}$ and adverse neonatal outcomes. NICU admission was significantly less likely as gestational age at delivery increased from 37 weeks to 39 completed weeks with rates of $7.3 \%$ at 37 weeks and $2.2 \%$ at 39 weeks, respectively ( $p$ for trend $<0.001$ ). Similar trends of decreasing incidence with higher gestational weeks were also observed for adverse respiratory outcomes (neonatal transient tachypnoea or RDS), neonatal infection, hypoglycaemia, hypoxic ischaemic encephalopathy, necrotising enterocolitis, hyperbilirubinaemia and prolonged hospitalisation. There were five neonatal deaths (two each at 38, 39 weeks and one at 40 weeks of gestation) and one intrapartum stillbirth associated with amniotic fluid embolism. We also assessed the neonatal outcomes for CD performed beyond 39 completed weeks of gestation. Compared with neonates born at 39 gestational weeks, there were significant trends towards an increased incidence of NICU admission for delivery at $\geq 40$ weeks of gestation $(p=0.011)$. Similar trends were noted for respiratory complications $(\mathrm{p}<0.001)$, hypoglycaemia $(\mathrm{p}<0.001)$, necrotising enterocolitis $(\mathrm{p}=0.003)$, hypoxic ischaemic encephalopathy $(\mathrm{p}=0.003)$ and prolonged neonatal hospitalisation $(\mathrm{p}=0.002)$.

The incidence of adverse maternal outcomes according to completed gestational week at $\mathrm{CD}$ is shown in online supplementary table 1 . There were no differences in maternal outcomes according to the timing of delivery.

The risks of adverse neonatal outcomes were decreased with increasing gestational age at term delivery up to 39 gestational weeks after adjusting for potential confounders (table 4). As compared with births at 39-39 6/7 weeks, births at 37-37 6/7 weeks were associated with an increased risk of adverse respiratory outcome (aOR: 4.82; $95 \%$ CI 3.35 to 6.94), neonatal infection (aOR: 3.68; 95\% CI 1.80 to 7.52), hypoglycaemia (aOR:3.85; 95\% CI 2.29 to 6.48 ), hyperbilirubinaemia (aOR: 3.50; 95\% CI 2.12 to 5.68), NICU admission (aOR: 3.73; 95\% CI 2.84 to 4.89 ) and prolonged hospitalisation (aOR: $7.51 ; 95 \%$ CI 5.10 to 11.07 ). Births at $38-386 / 7$ weeks, $40-406 / 7$ weeks or $\geq 41$ weeks were also associated

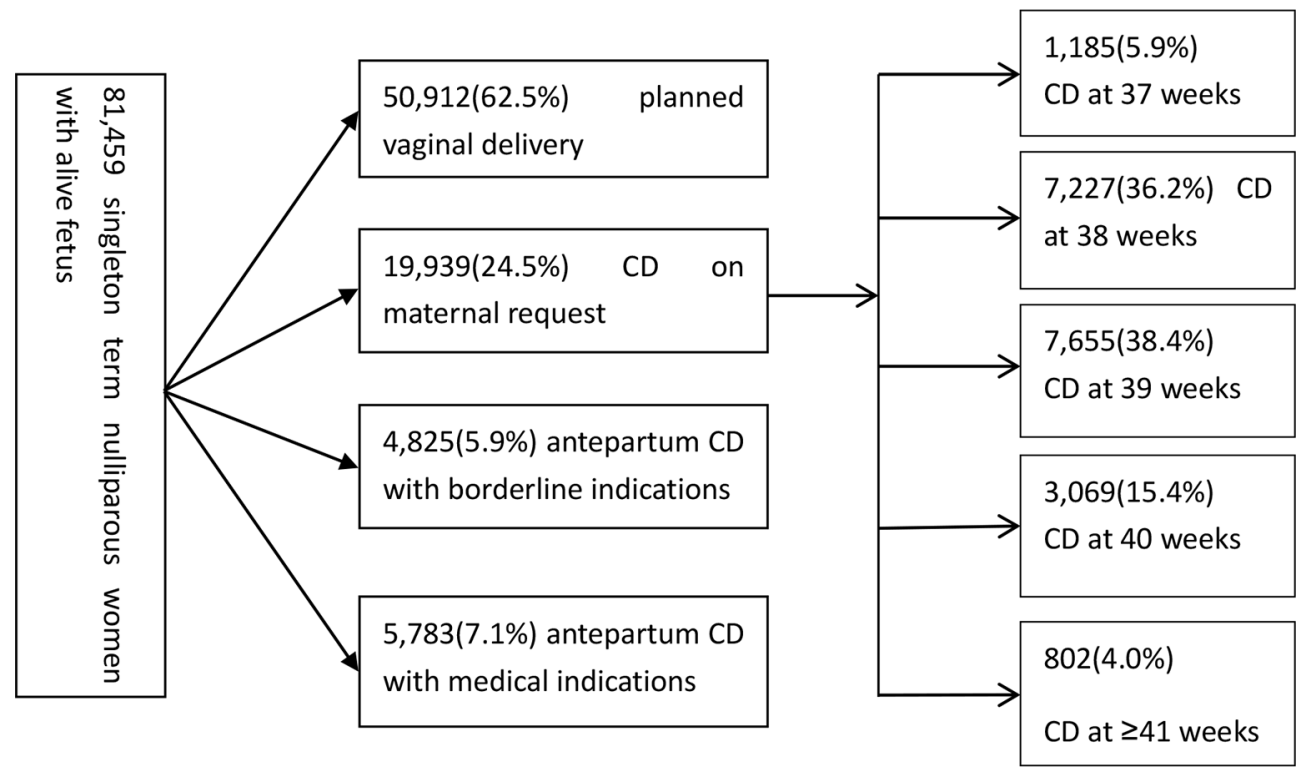

Figure 1 Flow chart of the study population. CD, caesarean delivery. 


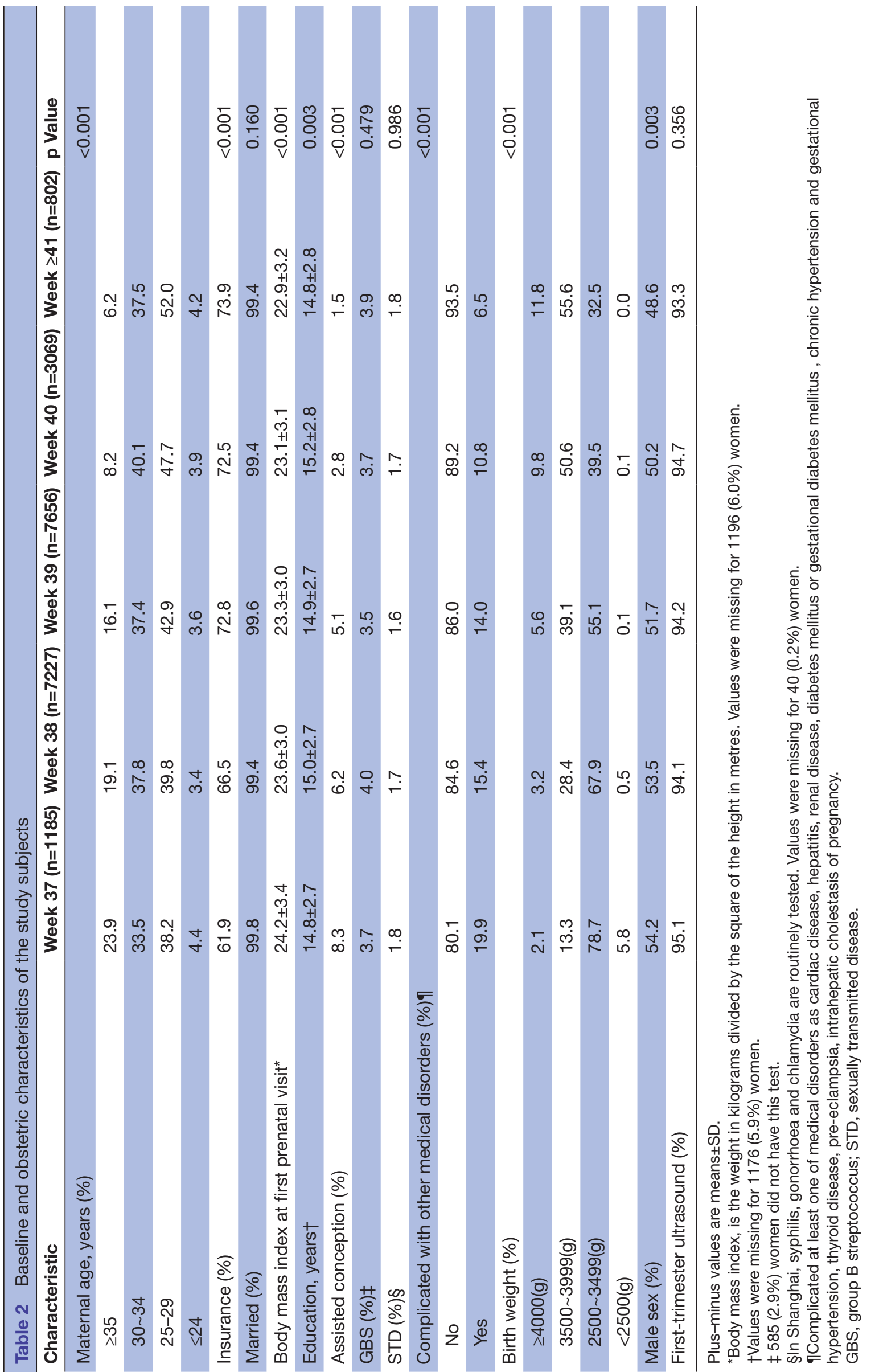




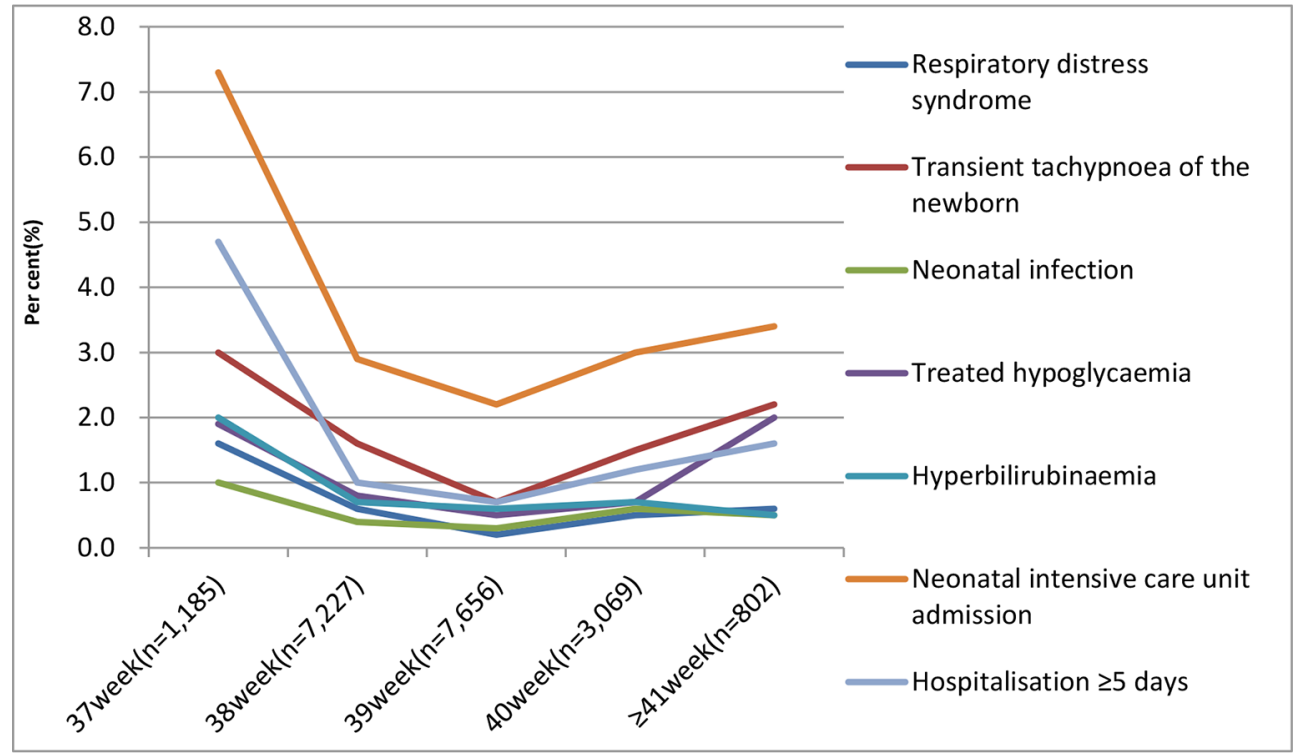

Figure 2 Timing of caesarean delivery and neonatal outcomes (International Peace Maternity \& Child Healthcare Hospital).

with an increased risk of adverse respiratory outcome with corresponding aORs $(95 \% \mathrm{CI})$ of $2.26(1.71-3.00), 1.97$ (1.33-2.94) and $2.91(1.80-4.70)$, respectively. Neonates born at these three gestational weeks were more likely to experience NICU admission (aOR: 1.38 (1.12-1.69), 1.37 (1.05-1.77) and 1.52 (1.00-2.31), respectively) and prolonged hospitalisation (aOR: 1.50 (1.05-2.15), 1.87 (1.23-2.86) and 2.46 (1.32-4.57), respectively).

\section{DISCUSSION}

This retrospective cohort study of antepartum elective or non-indicated CD at the largest obstetric centre in Shanghai, China demonstrates that compared with deliveries at 39 weeks, earlier deliveries were associated with a significantly increased risk of adverse neonatal outcomes. These included respiratory complications, neonatal infection, hypoglycaemia, hypoxic ischaemic encephalopathy, necrotising enterocolitis, hyperbilirubinaemia, NICU admission and prolonged hospitalisation to the NICU. Delivery $\geq 40$ weeks was also associated with increased risks of neonatal adverse outcomes.

The key strength of the present study is that it used a large hospital-based retrospective cohort data set which was published previously. Further, we performed a detailed examination of each woman's medical record such that the indication for CD could be clearly ascertained. IPMCHH's policy requiring a consent form for antepartum elective or non-indicated CD enabled us to determine truly non-medically indicated prelabour CDs. Confounding by indicated CD may limit the conclusions drawn from observational studies. In other such reports, the risk of an adverse outcome may be overestimated in $\mathrm{CD}$ undertaken prior to 39 complete weeks for some indications which might be associated with greater neonatal morbidity. We sought to eliminate this confounder by analysing cases of antepartum $\mathrm{CD}$ without indication. Important in the present study is the accuracy of gestational weeks. First-trimester ultrasound is routinely used to confirm gestational age in Shanghai, and $94.2 \%$ of pregnancies underwent first-trimester ultrasound in our study. Our study has some limitations as noted. First, the study population had a low body mass index and was very homogeneous (99\% Han) which strengthened our findings but may limit its generalisability to other populations with much higher rates of obesity in which perinatal risks of a caesarean may be appreciable. Additionally, the stillbirth rate might be different in a population with more obesity as discussed below. Second, there were only five neonatal deaths and one intrapartum stillbirth despite the large population studied, thus our study was underpowered to analyse the timing of CD in relation to these serious perinatal outcomes. Third, only women who successfully had elective $\mathrm{CD}$ at a certain gestational age were included and the women went into labour or emergency $\mathrm{CD}$ due to complications before the scheduled date might bias the results. However, it was reported less than $10 \%$ of women went into labour while waiting for delivery at 39 weeks in one clinical trial and the complications were extremely low in this low-risk population, so this bias is unlikely to be significant. ${ }^{16}$ Fourth, fetal lung maturity testing before elective early term delivery is not routinely used in China, thus we cannot be certain whether delivery $<39$ gestational weeks following a positive lung maturity test could reduce the neonatal morbidity prior to 39 weeks.

Our results are consistent with previous large size studies that performing elective $\mathrm{CD}<39+0$ weeks of gestation carries with it a significantly higher overall risk of various adverse neonatal outcomes. ${ }^{1-4}$ In contrast with Tita et $a l$ s and Wilmink et $a l$ s reports that found a higher risk of neonatal complications with $\mathrm{CD}$ at 41 weeks or later, ${ }^{13}$ our data showed a significantly higher risk for neonatal morbidity by postponing the caesarean section to $40+0$ weeks. This phenomenon could potentially be explained by ethnic differences as they relate to in utero pulmonary development. Patel et al reported the median gestational age of 


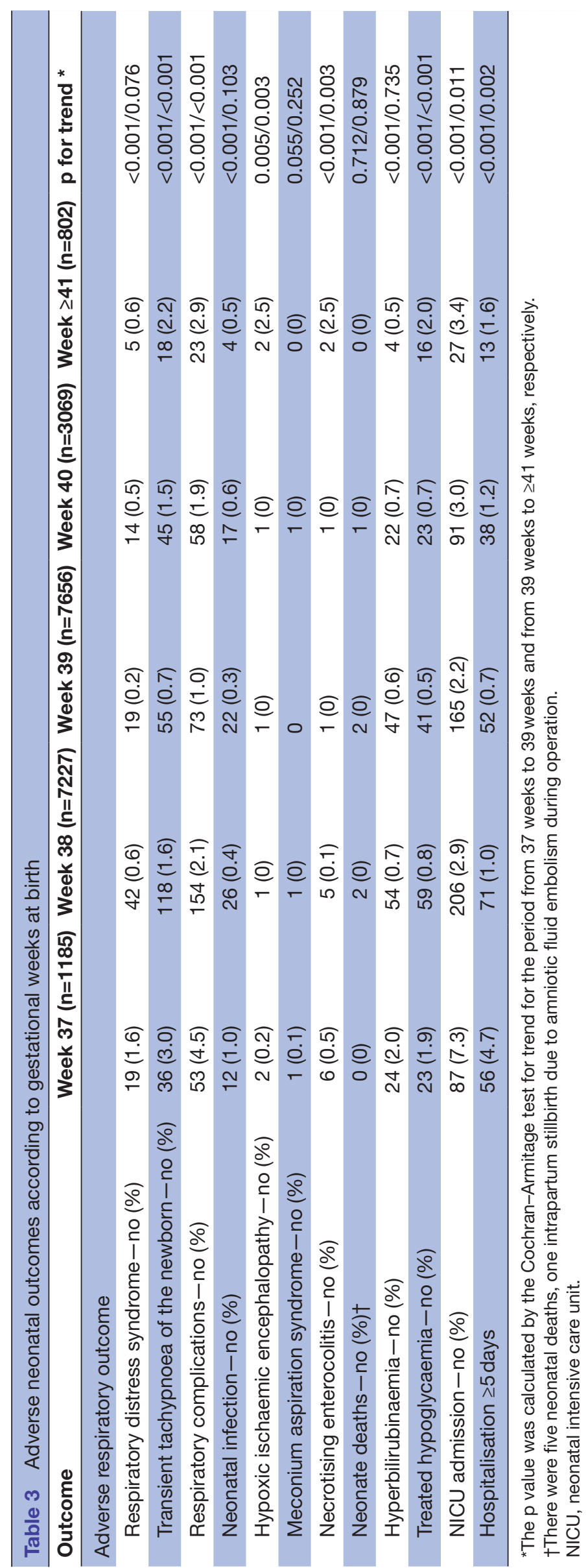




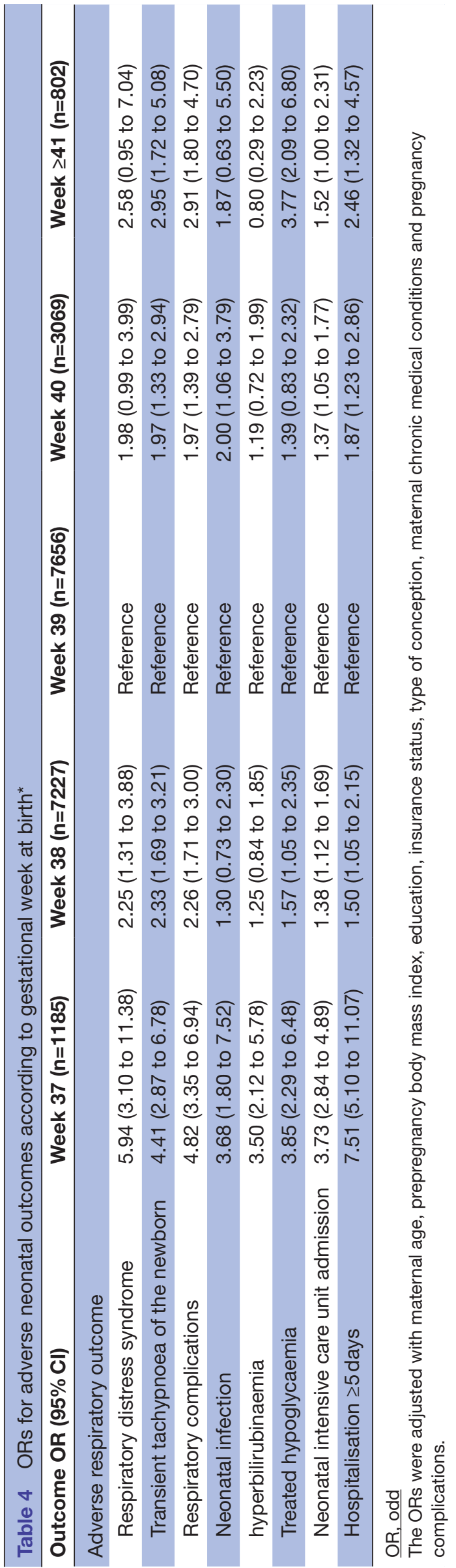

spontaneous delivery was 39 weeks in blacks and Asians and 40 weeks in white Europeans, ${ }^{9}$ thus fetal maturation may occur earlier in our population. So we speculate that $40+0$ weeks may be post-term for our population. A study compared delivery at each gestational age at term versus expectant management identified 39 weeks as the optimal timing of delivery, which also supports our findings. ${ }^{4}$

An important concern for optimal timing of elective delivery at term is the ongoing risk of stillbirth with increasing gestational weeks. In previous observational studies concerning optimal timing of elective caesarean section, the stillbirth rate was not included because of study design limitations. ${ }^{1-3}$ One report suggested that a policy limiting elective delivery before 39 weeks coincided with an increase in the risks of stillbirth at 37-38 gestational weeks. ${ }^{17}$ However, further studies of stillbirth trends based on the US population have not shown an association between increasing gestational weeks at term and stillbirth. ${ }^{18} 19$ In our study, we were able to calculate the stillbirth rates per 1000 ongoing pregnancies at each particular gestational week in our whole cohort. The stillbirth rates per 1000 ongoing pregnancies were $0.20,0.20,0.26,0.07$ and 0.16 at $37,38,39,40$ and 41 , respectively, which is lower than 0.2 of 1000 births at 37 completed weeks and 0.5 of 1000 births at 38 completed weeks among Scottish and Canadian populations. The lower stillbirth rate in our cohortng might be attributed to different local practices of antenatal surveillance in low-risk pregnancies such as included in the present study, also could be secondary to a lower BMI in our population since it is generally accepted that obesity is associated with an increased risk of stillbirth. ${ }^{20}$ It should be noted that we could only analyse the stillbirth rate in the whole cohort rather than the antepartum non-indicated CD group since many women with a stillbirth will have had a vaginal delivery after a stillbirth, thus making it impossible to distinguish between women who had a stillbirth while waiting for an elective $\mathrm{CD}$ or a planned vaginal delivery. Using the stillbirth rate of the entire cohort might therefore overestimate the stillbirth rate in our low-risk antepartum non-indicated CD population. On the basis of stillbirth rate in our population, we estimate 4-5 stillbirths every 10000 deliveries waiting from 37 weeks to 39 weeks. However, as compared with birth at 39 weeks, delivery at 37 weeks increased the rate of adverse infant outcomes including 140 extra cases of RDS, 51 necrotising enterocolitis, 70 neonatal infection, 16 hypoxic ischaemic encephalopathy, 510 admissions to the NICU regardless of the long-term adverse infant outcomes of early term births. ${ }^{21}{ }^{22}$ We also observed an increased risk of infant complications with $\mathrm{CD}$ at 40 weeks or later. Our results suggest that in addition to the risk of term stillbirth, the risk of neonatal complications may also be higher by postponing elective CD beyond 39-39 $6 / 7$ gestational weeks in our population.

Most studies of the timing of elective CD up to now are those of primarily repeat procedures, and other studies only include a small proportion of primary procedures 
such as only 788 cases of antepartum elective non-indicated $\mathrm{CD}$ in one study. ${ }^{2}$ Moreover, the primary procedures might be associated with medical and obstetric indications, which might bias the conclusion that elective $\mathrm{CD}$ should be performed beyond 39 gestational weeks. ${ }^{1-4} 23$ Our study importantly adds to the existing data on this subject and confirms the observation from other areas of the world that waiting until 39 weeks for elective CD is advisable. ${ }^{24}$ Since more than $25 \%$ of primary CDs are performed antepartum in other countries and even much higher in China, and since there is increasing enthusiasm for CDMR in western countries, the timing of primary $\mathrm{CD}$ and its effect on neonatal outcomes have substantial public health implications. ${ }^{1425}$

\section{Conclusion}

In summary, we demonstrated that elective caesarean section performed at 39-39 6/7 completed weeks of gestation was associated with better neonatal outcomes than earlier or later delivery in a Chinese population. The risk of stillbirth rate is low at term prior to 39 gestational weeks. CDMR should not be recommended, but for women who require elective $\mathrm{CD}$, neonatal outcome data suggest that delivery at 39 weeks is optimal timing.

Correction notice This paper has been amended since it was published Online First. Owing to a scripting error, some of the publisher names in the references were replaced with 'BMJ Publishing Group'. This only affected the full text version, not the PDF. We have since corrected these errors and the correct publishers have been inserted into the references.

Acknowledgements The authors thank Cheng Lei and Chen Ge, the information engineers of IPMCHH for their support of the data collection, and residents, fellows and the midwives in the labour and delivery room for the detailed delivery records.

Contributors YHU mainly contributed to design and critical writing. XL, WC and MBL mainly contributed to design, the critical revision and interpretation of the data, and the final approval of the version to be published. HS mainly contributed to the management of the cohort study and data collection.

Competing interests None declared.

Patient consent Detail has been removed from these case descriptions to ensure anonymity. The editors and reviewers have seen the detailed information available and are satisfied that the information backs up the case the authors are making.

Ethics approval The ethics review board at International Peace Maternity \& Child Healthcare Hospital. No:20140124.

Provenance and peer review Not commissioned; externally peer reviewed. Data sharing statement No additional data are available.

Open Access This is an Open Access article distributed in accordance with the Creative Commons Attribution Non Commercial (CC BY-NC 4.0) license, which permits others to distribute, remix, adapt, build upon this work non-commercially, and license their derivative works on different terms, provided the original work is properly cited and the use is non-commercial. See: http://creativecommons.org/ licenses/by-nc/4.0/

(c) Article author(s) (or their employer(s) unless otherwise stated in the text of the article) 2017. All rights reserved. No commercial use is permitted unless otherwise expressly granted.

\section{REFERENCES}

1. Tita AT, Landon MB, Spong CY, et al. Timing of elective repeat cesarean delivery at term and neonatal outcomes. $N$ Engl J Med 2009;360:111-20.

2. Hansen AK, Wisborg K, Uldbjerg N, et al. Risk of respiratory morbidity in term infants delivered by elective caesarean section: cohort study. BMJ 2008;336:85-7.

3. Wilmink FA, Hukkelhoven CW, Lunshof S, et al. Neonatal outcome following elective cesarean section beyond 37 weeks of gestation: a 7-year retrospective analysis of a national registry. Am J Obstet Gynecol 2010;202:250.e1-8.

4. Chiossi G, Lai Y, Landon MB, et al. Timing of delivery and adverse outcomes in term singleton repeat cesarean deliveries. Obstet Gynecol 2013;121:561-9.

5. American College of Obstetricians and Gynecologists. ACOG committee opinion no.561:nonmedically indicated early-term deliveries. Obstet Gynecol 2013;121:911-5.

6. Royal College of Obstetricians and Gynaecologists. Green-top guidelines. No. 7: antenatal corticosteroids to reduce neonatal morbidity and mortality. London: Royal College of Obstetricians and Gynaecologists, 2010.

7. Farrell S, Roye C, Crane J, et al. Statement on wait times in obstetrics and gynaecology. J Obstet Gynaecol Can 2008;30:248-57.

8. Balchin I, Whittaker JC, Lamont RF, et al. Timing of planned cesarean delivery by racial group. Obstet Gynecol 2008;111:659-66.

9. Patel RR, Steer P, Doyle P, et al. Does gestation vary by ethnic group? A London-based study of over 122,000 pregnancies with spontaneous onset of labour. Int J Epidemiol 2004;33:107-13.

10. Liu X, Landon MB, Cheng W, et al. Cesarean delivery on maternal request in China: what are the risks and benefits? Am J Obstet Gynecol 2015;212:817.e1-9.

11. Lumbiganon $P$, Laopaiboon M, Gülmezoglu $A M$, et al. Method of delivery and pregnancy outcomes in Asia: the WHO global survey on maternal and perinatal health 2007-08. Lancet 2010;375:490-9.

12. Zhang J, Liu Y, Meikle S, et al. Cesarean delivery on maternal request in southeast China. Obstet Gynecol 2008;111:1077-82.

13. Tang S, Li X, Wu Z. Rising cesarean delivery rate in primiparous women in urban China: evidence from three nationwide household health surveys. Am J Obstet Gynecol 2006;195:1527-32.

14. National Institutes of Health state-of-the-science conference statement: cesarean delivery on maternal request March 27-29, 2006. Obstet Gynecol 2006;107:1386-97.

15. Liu X, Lynch CD, Cheng WW, et al. Lowering the high rate of caesarean delivery in China: an experience from Shanghai. BJOG 2016;123:1620-8.

16. Glavind J, Kindberg SF, Uldbjerg N, et al. Elective caesarean section at 38 weeks versus 39 weeks: neonatal and maternal outcomes in a randomised controlled trial. BJOG 2013;120:1123-32.

17. Ehrenthal DB, Hoffman MK, Jiang X, et al. Neonatal outcomes after implementation of guidelines limiting elective delivery before 39 weeks of gestation. Obstet Gynecol 2011;118:1047-55.

18. MacDorman MF, Reddy UM, Silver RM. Trends in stillbirth by gestational age in the United States, 2006-2012. Obstet Gynecol 2015;126:1146-50.

19. Little SE, Zera CA, Clapp MA, et al. A multi-state analysis of earlyterm delivery trends and the association with term stillbirth. Obstet Gynecol 2015;126:1138-45.

20. Lindam A, Johansson S, Stephansson O, et al. High maternal body mass index in early pregnancy and risks of stillbirth and infant mortality-a population-based sibling study in Sweden. Am J Epidemiol 2016;184:98-105

21. Dong Y, Chen SJ, Yu JL. A Systematic review and meta-analysis of long-term development of early term infants. Neonatology 2012;102:212-21.

22. Moster D, Wilcox AJ, Vollset SE, et al. Cerebral palsy among term and postterm births. JAMA 2010;304:976-82.

23. Zanardo V, Simbi AK, Franzoi M, et al. Neonatal respiratory morbidity risk and mode of delivery at term: influence of timing of elective caesarean delivery. Acta Paediatr 2004;93:643-7.

24. Tita AT. Eunice Kennedy Shriver National Institute of Child Health and Human Development Maternal-Fetal Medicine Units Network. What we have learned about scheduling elective repeat cesarean delivery at term. Semin Perinatol 2016;40:287-90.

25. Meikle SF, Steiner CA, Zhang J, et al. A national estimate of the elective primary cesarean delivery rate. Obstet Gynecol 2005;105:751-6. 